\title{
Evaluación del efecto analgésico postoperatorio de infusiones intraoperatorias de tramadol y tramadol/lidocaína/ketamina en comparación con morfina/lidocaína/ketamina en hembras caninas sometidas a ovariohisterectomía
}

\author{
Evaluation of postoperative analgesic effect of intraoperative infusions of tramadol and \\ tramadol/lidocaine/ketamine compared with morphine/lidocaine/ketamine in \\ female dogs undergoing ovariohysterectomy
}

\author{
MA Fajardo ${ }^{\mathrm{a}}$, MA Lesmes ${ }^{\mathrm{a}}$, LA Cardona ${ }^{\mathrm{b}}$ \\ aUniversidad de La Salle, Bogotá, Colombia. \\ bUnidad de Cirugía Veterinaria, Facultad de Ciencias Agropecuarias, Programa de Medicina Veterinaria, \\ Centro de Investigación de la Interrelación de la Salud Animal, Humana y Ecológica (CISAHE), \\ Universidad de La Salle, Bogotá, Colombia.
}

\begin{abstract}
SUMMARY
The goal of this study was to evaluate and compare the post-operative analgesic efficacy of intra operative infusions of tramadol and tramadol/ lidocaine/ketamine compared to morphine/lidocaine/ketamine in female canines undergoing ovariohysterectomy. 30 female canines were used, without breed distinction with an average age between 1 and 10 years and weight between 2.5 y $37 \mathrm{kgs}$, creating 3 randomly allocated groups of 10 animals each: tramadol group, tramadol/lidocaine/ketamine group and morphine/lidocaine/ketamine group. All the animals were anesthetized using a standard technique and maintained with inhaled anesthetics, the surgical procedure was performed by the same trained surgeon. The postoperative analgesic effect was determined using the Melbourne Pain Scale, starting 30 minutes after endotracheal extubation, and every 30 minutes after that for a total data collection of 6 hours. The statistical analysis was performed with a Kruskal-Wallis test for non-parametric data. The results obtained noted that the pain scores from the tramadol group showed significant difference $(\mathrm{P}<0.05)$ at times $0.5,1.5,2$, 4 , y 5 hours evidencing an increase in the pain scores when compared to the morphine/lidocaine/ketamine group. The present study concluded that the association of multiple analgesics as a constant infusion provides a superior analgesic effect that the single use of an single infusion of tramadol in females canines undergoing ovariohysterectomy.
\end{abstract}

Palabras clave: analgesia postoperatoria, infusiones, tramadol.

Key words: postoperative analgesia, infusion, tramadol.

\section{INTRODUCCIÓN}

El reconocimiento, la cuantificación y el manejo del dolor en medicina veterinaria avanzan día a día, permitiendo que cada vez se realicen protocolos analgésicos más adecuados y seguros en los pacientes. En la actualidad las investigaciones en esta área se han ampliado, promoviendo el uso de nuevas herramientas: fáciles de usar, de aplicar y con mayor grado de efectividad, incrementando el interés y conciencia de la prevención y manejo del mismo.

La Asociación Internacional para el estudio del Dolor (IASP) define a este como aquella experiencia desagradable, emocional o sensitiva, asociada a una lesión de los tejidos, potencial o actual, descrita en términos de daño o lesión (Lamont y col 2000). Esta definición muestra que el dolor no sólo abarca un componente fisiopatológico, sino

Aceptado: 05.04.2012.

* Carrera 17 \# 19a -48 Sur, Barrio Restrepo, Bogotá, Colombia; lecardona@unisalle.edu.co también un componente emocional que en los animales es mucho más complejo de identificar y clasificar.

El estrés es una respuesta biológica, cuya función es tratar de mantener la homeostasis. Cuando esta respuesta no es efectiva aparece la enfermedad y el distrés; el cual lleva al dolor y sufrimiento del animal (Johnson y col 1992). El estrés y por lo tanto el dolor producen una serie de alteraciones a nivel cerebral, causando cambios fisiopatológicos en el animal. Hay una estimulación del sistema nervioso simpático que ocasiona cambios a nivel cardiorrespiratorio y digestivo (Hellyer y col 2007), se produce aumento o depresión de algunas hormonas, alteración de la función inmune, liberación de citoquinas proinflamatorias (Wiese y col 2005) y liberación de corticotropinas cerebrales las cuales van a llevar a cambios conductuales del animal, todo ello relacionado directamente con la intensidad del dolor (Muir 2002).

Debido a todos estos cambios fisiopatológicos, se han ampliado los campos de investigación en búsqueda de nuevos y eficaces tratamientos analgésicos. Razón por la cual se ha incursionado en el uso de las infusiones 
analgésicas continuas, ya que permiten conseguir un nivel constante de analgesia, evitan los picos de concentración en el plasma asociados a la administración de analgésicos intermitentes, como requieren de dosis menores disminuyen los efectos adversos de los medicamentos y permiten un mayor control sobre la administración del fármaco siendo de esta manera ideal en procedimientos quirúrgicos prolongados que requieren mayores niveles de analgesia. (Dyson 2008).

Entre los grupos de fármacos que se utilizan con mayor frecuencia en infusión analgésica se encuentran los opioides, en especial la morfina (Lucas y col 2001); los anestésicos locales, como la lidocaína, que por vía intravenosa provee analgesia peri y postoperatoria (Koppert y col 2004), y los antagonistas de receptores N-Metil-D-Aspartato no competitivos, como la ketamina (Muir y col 2003).

Ya que la morfina no es un medicamento de fácil acceso se sugiere el uso de otros medicamentos que presenten un efecto analgésico similar, con menos efectos secundarios y de fácil acceso como lo es el tramadol.

El objetivo de este estudio es evaluar y comparar el efecto analgésico postoperatorio de infusiones de tramadol solo y en combinación con lidocaína y ketamina con las infusiones de morfina/lidocaína/ketamina administradas durante el período intraoperatorio en hembras caninas sometidas a ovariohisterectomía.

\section{MATERIAL Y MÉTODOS}

\section{ANIMALES}

La clasificación de los pacientes se realizó mediante un examen clínico, el cual permitió hacer la clasificación de riesgo anestésico según la Sociedad de Anestesiólogos Americanos (ASA), utilizando para este estudio aquellos pacientes que fueron clasificados dentro de las categorías I y II.

Se utilizaron 30 caninos hembras procedentes de diferentes hogares de la ciudad de Bogotá-Colombia durante el año 2010. Los animales correspondieron a individuos de diferentes razas con un rango de peso entre 2.5 y $37 \mathrm{~kg}$ y un promedio de $19 \pm 10 \mathrm{~kg}$. Y un rango de edad entre 1 y 10 años con un promedio de $3 \pm 2$ años.

\section{PREPARACIÓN}

Como premedicación anestésica se utilizó acepromacina administrada a dosis de $0,05 \mathrm{mg} / \mathrm{kg}$ por vía intramuscular (Vaisanen y col 2002). Posteriormente se procedió a realizar la tricotomía del abdomen, de la cara medial del miembro posterior (derecho o izquierdo) y de la parte craneal del miembro anterior (derecho o izquierdo), en donde se colocó un catéter endovenoso 20 o 22 G en la vena cefálica correspondiente, a través del cual se administró cloruro de sodio $(\mathrm{NaCl}$ 0,9\%) a una velocidad de infusión de $7 \mathrm{ml} / \mathrm{kg} / \mathrm{hr}$ y en conjunto a través del mismo catéter se administraron las infusiones analgésicas preparadas en bolsas de $500 \mathrm{ml}$ con cloruro de sodio $(\mathrm{NaCl} \mathrm{0,9 \% )}$ a una velocidad de infusión de $3 \mathrm{ml} / \mathrm{kg} / \mathrm{hr}$ (Aguado y col 2010) para completar la dosis de fluidoterapia de mantenimiento de $10 \mathrm{ml} / \mathrm{kg} / \mathrm{hr}$ (Sarrau y col 2007).

Como inducción anestésica se utilizó tiopental sódico a una dosis de $7 \mathrm{mg} / \mathrm{kg}$ de peso administrado por vía intravenosa; y para el mantenimiento, se utilizó isoflurano mediante el empleo de un equipo de anestesia inhalatoria Surgivet $^{\circledR}$ (Máquina de anestesia inhalatoria. Modelo: University Anesthesia Machine V800100, USA) regulado al 0,5-2\% según las necesidades del paciente.

\section{TRATAMIENTOS}

La asignación del tratamiento analgésico intraoperatorio se realizó de manera aleatoria, conformando tres grupos de 10 animales cada uno: Al grupo tramadol se le administró una dosis de carga de $2 \mathrm{mg} / \mathrm{kg}$ de tramadol por vía intravenosa (Kukanich y Papich 2004), seguido de una infusión intravenosa continua de $22 \mu \mathrm{g} / \mathrm{kg} / \mathrm{min}$ de tramadol (Reza y col 2009). Al grupo tramadol/lidocaína/ketamina se le administró una dosis de carga de tramadol de $2 \mathrm{mg} / \mathrm{kg}$ intravenosa (Kukanich y Papich 2004), 1 mg/kg de lidocaína intravenosa y $0,25 \mathrm{mg} / \mathrm{kg}$ de ketamina intravenosa (Dyson 2008), seguido de una infusión intravenosa continua de $22 \mu \mathrm{g} / \mathrm{kg} / \mathrm{min}$ de tramadol (Reza y col 2009), $50 \mu \mathrm{g} / \mathrm{kg} / \mathrm{min}$ de lidocaína y $10 \mu \mathrm{g} / \mathrm{kg} / \mathrm{min}$ de ketamina (Muir y col 2003). Y finalmente al grupo morfina/lidocaína/ketamina se administró una dosis de carga de $0,5 \mathrm{mg} / \mathrm{kg}$ de morfina intravenosa lenta, $1 \mathrm{mg} / \mathrm{kg}$ de lidocaína intravenosa y $0,25 \mathrm{mg} / \mathrm{kg}$ de ketamina intravenosa (Dyson 2008), seguido de una infusión intravenosa continua de $3,3 \mu \mathrm{g} / \mathrm{kg} / \mathrm{min}$ de morfina, $50 \mu \mathrm{g} / \mathrm{kg} / \mathrm{min}$ de lidocaína y $10 \mu \mathrm{g} / \mathrm{kg} / \mathrm{min}$ de ketamina (Muir y col 2003). Todas las infusiones se usaron en bolsas de $500 \mathrm{ml}$ de cloruro de sodio $0,9 \%, 15$ minutos previos al comienzo de la cirugía, el cual se determinó en el momento en que el cirujano realizó una incisión por la línea media ventral del paciente y finalizaron en el momento en el que se realizó el último punto en piel. El cirujano fue el mismo para todos los procedimientos quirúrgicos con conocimiento del tratamiento instaurado en cada paciente.

\section{EVALUACIÓN ALGÉSICA}

Una vez finalizada la cirugía, los pacientes fueron trasladados a la sala de recuperación y 30 minutos posteriores a la extubación endotraqueal se inició la evaluación analgésica postoperatoria, la cual se realizó cada 30 minutos hasta completar un período de seis horas; ésta fue realizada por un evaluador capacitado, el cual desconocía los tratamientos farmacológicos intraoperatorios.

El método usado para la determinación de grado de algesia se basó en la escala de dolor de la Universidad de Melbourne. Esta escala establece seis categorías que 
incluyen información sobre variables fisiológicas (dilatación pupilar, frecuencia cardiaca, frecuencia respiratoria, temperatura rectal y salivación) y comportamentales como respuesta a la palpación, actividad, estado mental, postura y vocalización (Firth y Haldane 1999).

Las variables son evaluadas con opciones de respuesta numeradas que van desde el 0 hasta el 3 , indicando el grado de dolor. Así 0 indica la ausencia de dolor, 1 dolor leve, 2 dolor moderado y 3 dolor severo. La suma de puntajes de todas las variables podrá dar un total de hasta 24 puntos (Morton y col 2005). El total de puntos definirá el grado de dolor del paciente (Hellebrekers 2002) de la siguiente manera: el valor total igual a 0 puntos indica ausencia de dolor, entre 1 y 8 puntos indica dolor leve, entre 9 y 15 puntos indica dolor moderado y entre 16 y 24 puntos dolor severo (Bravo y col 2008). Si el paciente mostró una puntuación por encima de 9 durante más de dos evaluaciones consecutivas se suministró un analgésico de rescate, butorfanol a razón de $0,4 \mathrm{mg} / \mathrm{kg}$ IM (Dyson 2008), sin embargo esto no fue necesario para ningún paciente.

Las variables se evaluaron clasificándolas en tres grupos: puntaje fisiológico, puntaje comportamentales y puntaje final que corresponde a la sumatoria de las dos anteriores.

\section{ANÁLISIS ESTADÍSTICO}

El diseño del presente trabajo se realizó con tres grupos y utilizó un diseño completamente aleatorio con diez repeticiones para cada tratamiento farmacológico.

Los datos obtenidos se registraron y anotaron en la planilla correspondiente para estos efectos que estuvo realizada en una hoja de cálculo electrónico tipo Excel, para ser posteriormente tabulados en el programa estadístico Statistix 8.0.

La normalidad de la distribución de los datos se midió utilizando el test de Shapiro-Wilk y la homocedasticidad de las varianzas entre cada uno de los tratamientos fue evaluada mediante el test de Bartlett.

A los datos no paramétricos se les realizó un test de Kruskal-Wallis de una vía y posteriormente un test de Dunn para evaluar la diferencia entre grupos de datos. Se consideraron estadísticamente significativos valores $\mathrm{P}<0,05$. Los resultados se expresaron en su mediana y cuartiles 1 y $3\left(Q_{1}\right.$ y $\left.Q_{3}\right)$.

Se elaboraron las figuras y tablas en Microsoft Excel y los datos postoperatorios se presentaron como medianas.

\section{RESULTADOS}

Con el fin de evaluar el grado de dolor postoperatorio mediante la escala de dolor de la Universidad de Melbourne, se definió el tiempo 0,5 como el momento inicial en el que se comenzaron a registrar los distintos parámetros postextubación del paciente a intervalos de 30 minutos.
PUNTAJE FINAL

El valor mediana para el grupo tramadol fue de $4(2,6)$ puntos de grado de dolor, para el grupo tramadol/lidocaína/ketamina de $2(1,3)$ puntos y para el grupo morfina/ lidocaína/ketamina de $1(0,2)$ punto.

Los grados de dolor durante los tiempos 0,$5 ; 1,5 ; 2 \mathrm{y}$ 5 para el grupo tramadol obtuvieron valores entre 3 y 7,5 puntos los cuales fueron mayores que en el grupo morfina/ lidocaína/ketamina que estuvieron entre 0,5 y 2,5 puntos, siendo las diferencias estadísticamente significativas $(\mathrm{P}<0,05)$. Sin embargo, el grupo tramadol/lidocaína/ ketamina no mostró diferencias estadísticamente significativas con respecto a los grupos tramadol y morfina/ lidocaína/ketamina.

En el tiempo 3 de evaluación postoperatoria el grupo tramadol obtuvo una puntación de 4 siendo éste mayor al puntaje obtenido por los grupos tramadol/lidocaína/ketamina y morfina/lidocaína/ketamina con una puntación de 1 , siendo estas diferencias estadísticamente significativas $(\mathrm{P}<0,05)$.

En los tiempos $1 ; 2,5 ; 3,5 ; 4 ; 4,5 ; 5,5 ; 6$ y 6,5 los tres grupos mostraron es sus puntajes finales de la escala de Melbourne un comportamiento similar, con rangos de dolor entre 0,5 y 6 sin ser las diferencias estadísticamente significativas $(\mathrm{P}>0,05)$ entre ellos (figura 1$)$.

Puntaje fisiológico. El valor mediana para el grupo tramadol fue de $1(0,2)$ punto de grado de dolor, para el grupo tramadol/lidocaína/ketamina de $1(0,1)$ y para el grupo morfina/lidocaína/ketamina de $0(0,0)$ puntos.

Los grados de dolor durante los tiempos $1 ; 1,5 ; 2 ; 3 \mathrm{y}$ 3,5 para el grupo tramadol mostraron valores entre 1,5 y 2 puntos los cuales fueron mayores al grupo morfina/ lidocaína/ketamina que se mantuvieron en 0 puntos, siendo estadísticamente significativos $(\mathrm{P}<0,05)$. Sin embargo, el grupo tramadol/lidocaína/ketamina se comportó de manera similar al grupo tramadol y morfina/lidocaína/ ketamina.

En los tiempos 0,$5 ; 2,5$ y a partir de las cuatro horas de evaluación postoperatoria los tres grupos mostraron en los puntajes finales de la categoría fisiológica de la escala de Melbourne un comportamiento similar, con rangos de dolor entre 0 y 4 sin ser estadísticamente significativos $(\mathrm{P}>0,05)$ entre los grupos (figura 2$)$.

Puntaje comportamental. El valor mediana para el grupo tramadol fue de $2(1,4)$ puntos de grado de dolor, para el grupo tramadol/lidocaína/ketamina y para el grupo morfina/ lidocaína/ketamina de $1(0,2)$ punto.

Al evaluar los puntajes totales de la categoría comportamental en la escala de Melbourne se observó que entre los tratamientos evaluados mostraron un comportamiento similar, con rangos de dolor entre 0,5 y 2,5 sin ser estadísticamente significativas $(P>0,05)$ entre ellos (figura 3 ). 


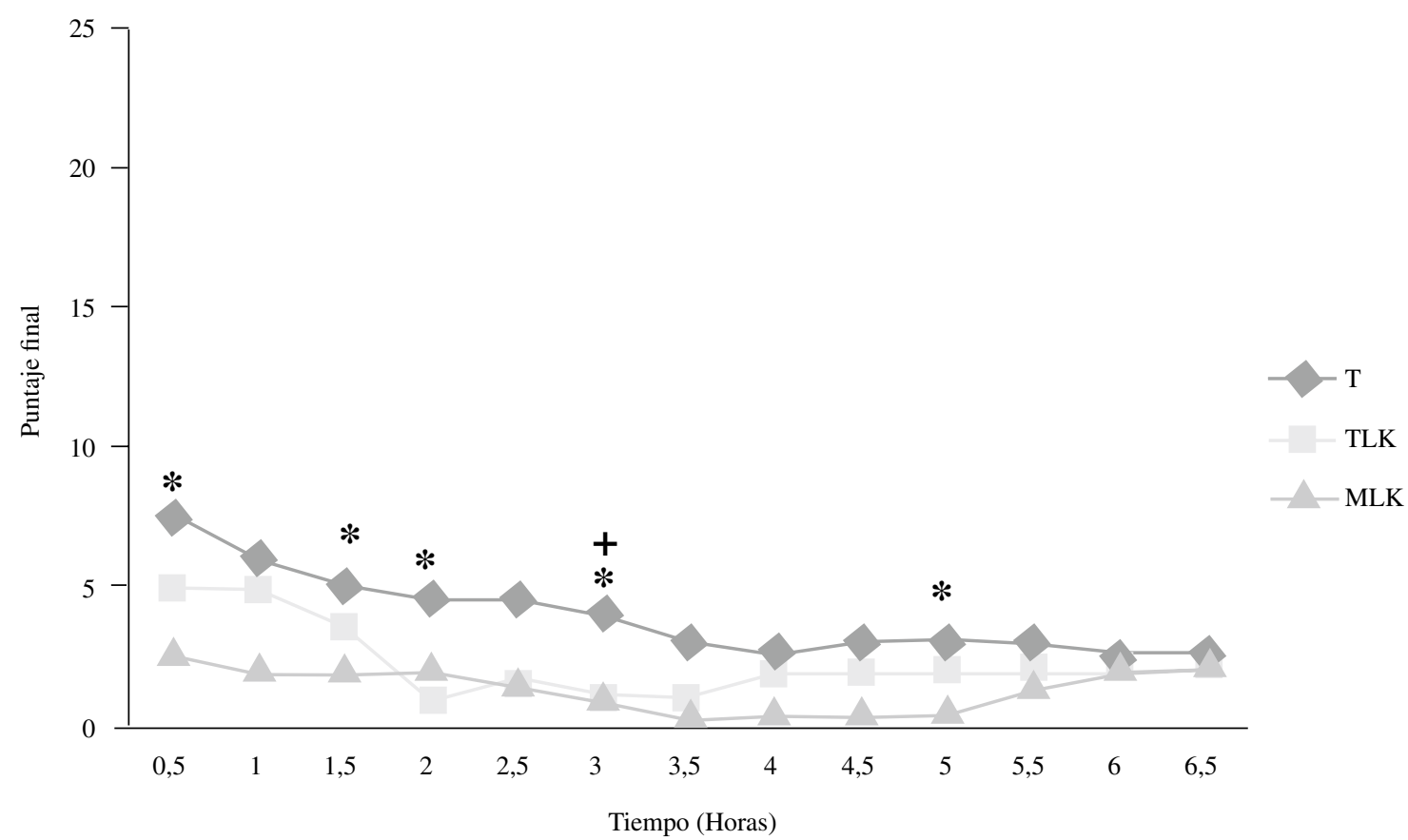

Figura 1. Mediana de los puntajes finales de dolor postquirúrgico en la escala de Melbourne. Para los grupos tramadol (T), tramadol/lidocaína/ketamina (TLK) y morfina/lidocaína/ketamina (MKL) durante la evaluación del efecto analgésico postoperatorio de infusiones intraoperatorias en hembras caninas sometidas a ovariohisterectomía $(\mathrm{n}=30)$.

Median of finally postoperative pain scores in the Melbourne scale. For tramadol, tramadol/lidocaine/ketamine and morphine/lidocaine/ketamine groups during the assessment of postoperative analgesic effect of intraoperative infusion in female dogs undergoing ovariohysterectomy $(\mathrm{n}=30)$.

* Diferencias estadísticamente significativas entre los grupos tramadol y morfina/lidocaína/ketamina $(\mathrm{P}<0,05)$.

* Statistically significant difference between tramadol group and morphine/lidocaine/ketamine group $(\mathrm{P}<0,05)$.

+ Diferencias estadísticamente significativas entre los grupos tramadol y tramadol/lidocaína/ketamina $(\mathrm{P}<0,05)$.

$+\quad$ Statistically significant difference between tramadol group and tramadol/lidocaine/ketamine group $(\mathrm{P}<0.05)$.

\section{DISCUSIÓN}

El reconocimiento y la cuantificación del dolor en los animales son complejos por su incapacidad para comunicarse y la respuesta individual en la manifestación del dolor. Por esta razón el uso de puntuaciones de dolor es útil para evaluar la eficacia de una técnica analgésica. La escala ideal debe ser objetiva, fiable y fácil de usar. Hasta la fecha los sistemas de puntuación de dolor usan una variedad de escalas, entre las que se encuentran: la escala visual análoga, la escala numérica, la escala descriptiva simple, escala de la Universidad de Melbourne, entre otras. Desafortunadamente todas son subjetivas, incluso si usan la medición de variables fisiológicas (Holton y col 1998, Firth, Haladane 1999).

En el presente estudio se utilizó como método de evaluación analgésica postoperatoria una escala de respuestas fisiológicas y comportamentales, tal y como lo sugiere Holton y col (2001) y Morton y col (2005), conocida como la escala de dolor de la Universidad de Melbourne. La principal ventaja que tiene esta escala con respecto a las escalas de tipo subjetivo es una mayor precisión al otorgar mayor valor a ciertos parámetros con respecto a otros. Como es una escala que evalúa múltiples factores, incrementa su sensibilidad y especificidad. Sin embargo, limita la validación de resultados, ya que no detecta pequeñas variaciones nociceptivas especialmente si las observaciones son realizadas esporádicamente (Hellyer 2002). Esta escala permitió mostrar un nivel bajo de dolor en los tres grupos evaluados, mostrando medianas promedio entre 1 y 4 grados de dolor, en una escala del 0 al 24 lo cual corresponde a un buen nivel analgésico.

La principal acción farmacología descrita del tramadol y la morfina es la analgesia. El clorhidrato de tramadol (1RS, 2RS)-2-[(dietilamin)-metil)-1-(3metoxifenil)-ciclohexanol HCL], es un análogo de la codeína que actúa a nivel central, su efecto analgésico es definido por su acción opioide leve con selectividad para receptores $\mu$ y por su interferencia con la recaptación de serotonina y norepinefrina en los tractos descendentes inhibitorios (Raffa y col 1993). Este doble mecanismo de acción se puede atribuir a los dos enantiómeros de tramadol racémico. El enantiómero (+) tiene una mayor afinidad por los receptores $\mu$ y por la inhibición de la recaptación de serotonina, mientras que el enantiómero (-) es un inhibidor más eficaz de la recaptación de noradrenalina (Scott y Perry 2000). Por 


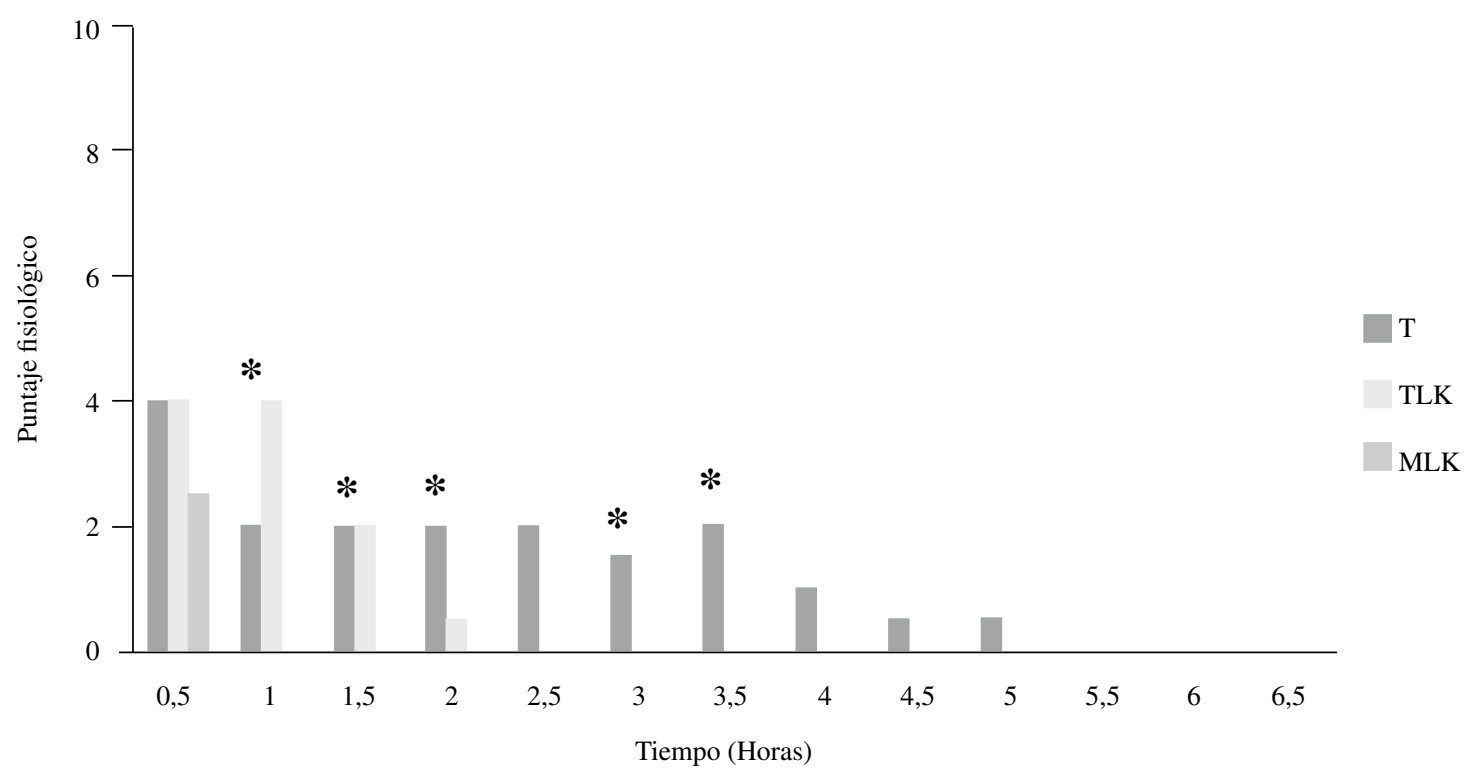

Figura 2. Mediana de los puntajes de dolor postquirúrgico en el parámetro fisiológico de la escala de Melbourne. Para los grupos tramadol (T), tramadol/lidocaína/ketamina (TLK) y morfina/lidocaína/ketamina (MLK) durante la evaluación del efecto analgésico postoperatorio de infusiones intraoperatorias en hembras caninas sometidas a ovariohisterectomía $(\mathrm{n}=30)$.

Median of postoperative pain scores in the physiological parameter of the Melbourne scale. For tramadol, tramadol/ lidocaine/ketamine and morphine/lidocaine/ketamine groups during the assessment of postoperative analgesic effect of intraoperative infusion in female dogs undergoing ovariohysterectomy $(\mathrm{n}=30)$.

Diferencias estadísticamente significativas entre los grupos tramadol y morfina/lidocaína/ketamina $(\mathrm{P}<0,05)$.

* Statistically significant difference between tramadol group and morphine/lidocaine/ketamine group $(\mathrm{P}<0.05)$.

su parte la morfina es un agonista opioide que ejerce su efecto analgésico gracias a la estimulación de receptores $\mu, \kappa$ y $\delta$ a nivel periférico, médula espinal y estructuras supraespinales (Lamont y col 2000).

El grupo morfina/lidocaína/ketamina fue el que produjo un comportamiento más estable y eficaz durante las seis horas de evaluación postoperatoria, mostrando un valor de mediana promedio menor que los otros dos grupos; este efecto analgésico es definido por Raposo y col (2009), quienes concluyen que los opioides son los fármacos con mayor eficacia analgésica de cuantos se conocen, debido a que este efecto es el resultado de una acción combinada sobre estructuras situadas a diversos niveles del neuroeje, interviniendo tanto en mecanismos aferentes como eferentes de la sensibilidad nociceptiva. Kukanich y Papich (2004) reportan que las infusiones continuas intravenosas con morfina han sido usadas con éxito en perros.

El efecto analgésico postoperatorio del tramadol y la morfina es también descrito por Kongara, y col (2009), quienes en su estudio en perros demostraron que aunque el efecto analgésico postoperatorio del tramadol fue menor al de la morfina, no hubo diferencias estadísticamente significativas entre los umbrales nociceptivos de ambos grupos durante una hora de evaluación postoperatoria. Nolan (2002) corrobora estos resultados reportando un menor efecto analgésico del tramadol ya que su afinidad por los receptores $\mu$ es 6000 veces menor que el de la morfina. Sin embargo, Yazbek y Fantoni (2005) demostraron en un estudio realizado con 15 perros que iban a ser sometidos a procedimientos quirúrgicos ortopédicos que el tramadol puede utilizarse con éxito para el dolor postoperatorio. En otro estudio el tramadol mostró una eficacia equivalente a la morfina administrado preoperatoriamente en pacientes caninos que fueron sometidos a ovariohisterectomía (OVH) (Mastrocinque y Fantoni 2003). Este mismo resultado se obtuvo en un estudio realizado por Vickers y Paravicini (1995), quienes compararon el efecto analgésico postoperatorio en pacientes que fueron sometidos a cirugía abdominal.

Por otro lado el efecto analgésico postoperatorio de la lidocaína y la ketamina que se evidenció en el presente estudio es también descrito por varios autores. Koppert y col (2004) describen la lidocaína como una aminoamida perteneciente al grupo de anestésicos locales la cual proporciona analgesia cuando es administrada por vía intravenosa. Su efecto analgésico se debe al bloqueo de canales individuales de sodio en las fibras nerviosas sensoriales, lo cual inhibe la conducción de impulsos eléctricos. Anis y col (1983) describen la ketamina como un análogo de la feniciclidina a la cual se le ha descrito un efecto analgésico, este efecto se atribuye a que impide la exagerada respuesta y actividad (sensibilización central) de todo el rango dinámico de las neuronas en el asta dorsal de la médula espinal de los estímulos nociceptivos que se 


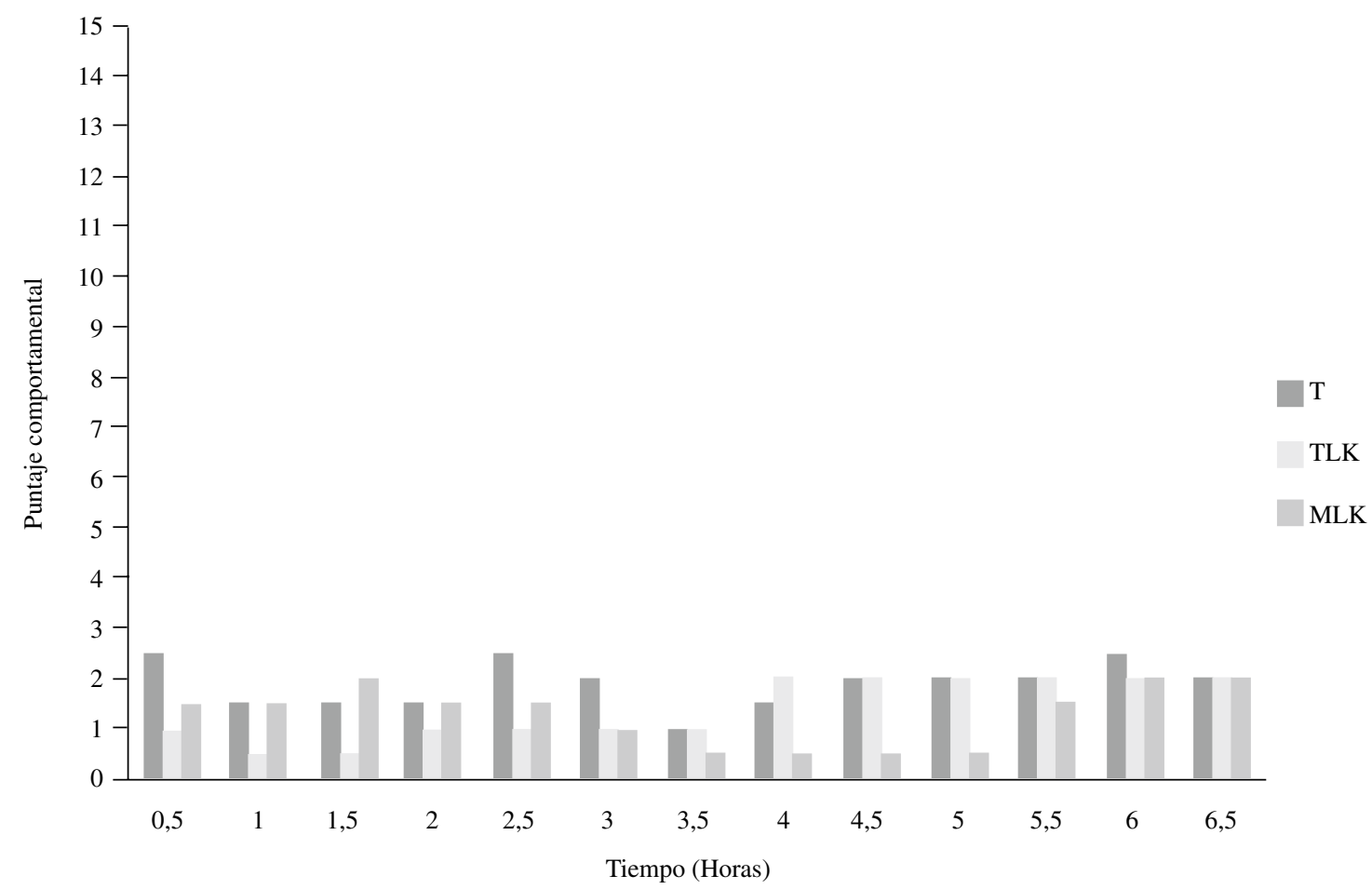

Figura 3. Mediana de los puntajes de dolor postquirúrgico en el parámetro comportamental de la escala de Melbourne. Para los grupos tramadol (T), tramadol/lidocaína/ketamina (TLK) y morfina/lidocaína/ketamina (MLK) durante la evaluación del efecto analgésico postoperatorio de infusiones intraoperatorias en hembras caninas sometidas a ovariohisterectomía $(\mathrm{n}=30)$.

Median of postoperative pain scores in the behavioral parameter of the Melbourne scale. For tramadol, tramadol/lidocaine/ketamine and morphine/lidocaine/ketamine groups during the assessment of postoperative analgesic effect of intraoperative infusion in female dogs undergoing ovariohysterectomy $(\mathrm{n}=30)$.

llevan a cabo por las neuronas aferentes del dolor, actuando como un antagonista no competitivo de los receptores N-Metil-D-Aspartato, de igual forma Pekoe y Smith (1982) han descrito efectos analgésicos pro agonismo de receptores opioides y activación parcial de las neuronas serotoninergicas y noradrenergicas del sistema nervioso central. Wagner y col (2002) manejaron como analgesia en caninos sometidos a amputación de la extremidad anterior; infusiones de ketamina, dando como resultado puntajes analgésicos postoperatorios menores a las $12 \mathrm{y}$ 18 horas después de la cirugía al ser comparados con el grupo placebo. Smith y col (2004) demostraron en un estudio piloto en perros sometidos a cirugía intraocular puntajes analgésicos postoperatorios similares entre las infusiones intravenosas de lidocaína y morfina.

\section{PARÁMETROS FISIOLÓGICOS}

El dolor inducido produce una serie de respuestas fisiológicas que se traducen en un aumento del tono simpático que se manifiesta como, vasoconstricción, aumento del gasto cardiaco a través de incrementos en el volumen sistólico y la frecuencia cardiaca y aumentos del trabajo del miocardio a través de incrementos en la tasa metabólica y consumo de oxígeno (Hellyer y col 2007). Es por esta razón que el conocimiento de los procesos fisiológicos asociados al dolor permite un diagnóstico correcto del mismo, en muchos casos permite evaluar su intensidad, deduciendo de esta forma la eficacia del analgésico suministrado (Flecknell y Waterman-Pearson 2000). Sin embargo, es indispensable conocer los diferentes efectos de los analgésicos en los parámetros fisiológicos del paciente. Por ejemplo los analgésicos opioides están asociados con depresión respiratoria, mediada a través de una disminución en la sensibilidad del centro respiratorio del $\mathrm{CO}_{2}$ lo cual se traduce en una disminución de la frecuencia respiratoria y el volumen tidal (Scott y Perry 2000).

En el presente estudio el puntaje fisiológico fue influenciado mayoritariamente por las variables frecuencia cardiaca (FC) y frecuencia respiratoria (FR). Ya que durante las primeras 3,5 horas de evaluación analgésica postoperatoria se observaron puntajes mayores en estas variables en el grupo tramadol, siendo estos cambios estadísticamente significativos ( $\mathrm{P}<0,05)$ a los presentados por el grupo morfina/lidocaína/ ketamina. Lo cual es explicado por Lamont y col (2000) quienes describen que un enfoque multimodal para el manejo del dolor proporciona una analgesia superior. Después de las 3,5 horas de evaluación analgésica postoperatoria la FC 
y FR no mostraron cambios estadísticamente significativos $(\mathrm{P}>0,05)$ en ninguno de los tres grupos. Esto coincide con lo reportado por Mastrocinque y Fantoni (2003), quienes demostraron en un estudio comparativo de morfina y tramadol en hembras sometidas a OVH, con seis horas de evaluación postoperatoria, valores de FC y FR sin cambios estadísticamente significativos para ambos grupos. McMillan y col (2008), en un estudio en el que se administraban dosis intravenosas de tramadol de $1 ; 2$ y $4 \mathrm{mg} / \mathrm{kg}$ en perros, demostraron la ausencia de depresión cardiaca y respiratoria y el incremento de la salivación y náuseas después de cada dosis de tramadol. Coincidiendo de igual forma con que el mayor número de animales que presentaron salivación formaban parte del grupo tramadol seguido del grupo tramadol/lidocaína/ketamina; además el único animal que durante el presente estudio presentó vómito formaba parte del grupo tramadol. En humanos el vómito y las nauseas han sido reportados después de una rápida administración intravenosa, recomendando una administración intravenosa lenta durante 1 a 2 minutos (Lewis y Han 1996). McMillan y col (2008) reportan una disminución de estos efectos adversos con el uso de tramadol en infusión.

El grupo morfina/lidocaína/ketamina mostró a partir de la primera hora de evaluación analgésica postoperatoria, en los parámetros fisiológicos, un completo nivel analgésico, que se manifestó hasta las seis horas de evaluación analgésica una mediana promedio de 0 . Puntaje que se corresponde, en la escala de dolor de la Universidad de Melbourne a un paciente sin dolor. Otero (2004) reporta que un efecto constante para los agonistas $\mu$ es la disminución en la FC, pero que este efecto cardiovascular generalmente disminuye al disminuir la dosis empleada. Al igual que Hall y col (2001), quienes reportan que a dosis bajas la morfina tiene mínimos efectos cardiovasculares.

Uno de los parámetros fisiológicos que maneja la escala de dolor de la Universidad de Melbourne es la dilatación pupilar. En el grupo morfina/lidocaína/ketamina este parámetro a partir de las dos horas de evaluación postoperatoria no se vio afectado ya que la totalidad del grupo evidenció miosis. La mayoría de los opioides causan constricción pupilar, la cual es consecuencia de la acción de estos sobre el núcleo Edinger-Westphal, dando lugar a la estimulación del segmento autónomo del nervio oculomotor (Borsook y col 1999).

La temperatura fue otro de los parámetros fisiológicos evaluados por la escala del dolor de la Universidad de Melbourne. Este parámetro no se vio afectado en ninguno de los tres grupos durante las seis horas de evaluación, debido a que en todos los grupos se presentó hipotermia. Los opioides pueden cambiar los puntos de equilibrio de los mecanismos hipotalámicos reguladores de la temperatura, y normalmente producen un ligero descenso de la temperatura (Borsook y col 1999). El maleato de acepromacina usado en este estudio como premedicación en los tres grupos se le atribuye una importante reducción de temperatura corporal tal y como lo reportan Caballero y Ahumada (2002). La anestesia en general disminuye la tasa metabólica basal entre un 15 y $44 \%$ inhibiendo la actividad muscular, lo que produce disminución en la producción de calor. Agentes anestésicos como el isoflurano aumentan el umbral de termorregulación, producen vasoconstricción periférica generando redistribución central del calor; además de esto, la intubación impide el calentamiento nasal del aire inspirado, la aplicación de antisépticos aumenta la pérdida de calor a medida que se evaporan y el frío de la superficie de la mesa provoca pérdidas por conducción (Oncken y col 2001).

\section{PARÁMETROS COMPORTAMENTALES}

Aunque todos los animales experimentan dolor, la expresión del dolor varía con la edad y especie, así como entre los individuos. Las respuestas a la cirugía, lesión o tratamiento son exclusivas de cada individuo y las diferencias reflejan la variación genética en factores como el número, distribución y morfología de los receptores opioides (Hellyer 2002).

En la presente investigación la observación de la conducta animal fue indispensable para deducir la ausencia de dolor. Lo que concuerda con lo reportado por Hellebrekers (2002) en donde los investigadores que trabajan en diversos métodos de evaluación del dolor concluyen que la observación de la conducta animal es un parámetro útil para distinguir la ausencia del dolor o la presencia de dolor moderado a intenso.

Al evaluar la respuesta a la palpación, se obtuvieron las puntuaciones más elevadas en el grupo tramadol, siendo este un valor representativo en el incremento de puntaje de dolor total. Un estudio realizado por Wiese y col (2005) concluyó que los signos de dolor más común en los perros son renuencia a levantarse o moverse y dolor a la palpación de la zona afectada, lo que se pudo confirmar con los resultados obtenidos en el grupo tramadol. Lascelles (2000) concluyó con respecto a la evaluación de dolor en los animales, que la simple observación es un método inadecuado de valoración, lo que coincide con nuestro estudio, ya que el puntaje de dolor total se vio influenciado en mayor medida por los parámetros fisiológicos y a nivel comportamental por la respuesta a la palpación como se mencionó con anterioridad.

$\mathrm{Al}$ evaluar la categoría de postura, se observó que las más frecuentes en los tres grupos eran la recumbencia lateral seguida de la esternal. Lo que coincide con los resultados obtenidos por Hardie y col (1997), quienes establecieron el comportamiento postoperatorio de los perros enjaulados después de ser sometidos a OVH, en donde concluyeron a las seis horas de evaluación comportamental postoperatoria que los perros pasaban significativamente más tiempo dormidos y en recumbencia lateral.

Del presente estudio se puede concluir que para hacer una adecuada evaluación algésica postoperatoria es indispensable una evaluación de parámetros fisiológicos y 
comportamentales. La escala de dolor de la Universidad de Melbourne permite hacer dicha evaluación, aunque se requiere hacer una identificación previa de los efectos que pueden producir los analgésicos suministrados, ya que pueden interferir en algunos de los parámetros evaluados y por lo tanto estos no serian útiles como parámetros evaluadores de dolor.

Finalmente, con base a la respuesta algésica presentada en el período postoperatorio se concluye que a las dosis estudiadas, la asociación de varios analgésicos en infusión, proveen mayor efecto analgésico que el uso de tramadol solo en infusión, observándose disminución y en muchos casos ausencia de los efectos secundarios de cada uno de ellos. Sin embargo, los tres protocolos analgésicos son eficaces para el control del dolor agudo en hembras caninas sometidas a ovariohisterectomía.

\section{RESUMEN}

El objetivo de este estudio fue evaluar y comparar la eficacia analgésica postoperatoria de infusiones intraoperatorias de tramadol y tramadol/lidocaína/ketamina en comparación con morfina/lidocaína/ ketamina en hembras caninas sometidas a ovariohisterectomía. Se usaron 30 hembras caninas sin distinción de raza con una edad entre 1 y 10 años y un peso entre 2,5 y 37 kilogramos, conformando tres grupos aleatorios de 10 animales cada uno: grupo tramadol, grupo tramadol/lidocaína/ ketamina y grupo morfina/lidocaína/ketamina, bajo anestesia general inhalada y ovariohisterectomía realizada por un mismo cirujano para todos los pacientes. El efecto analgésico postoperatorio se determinó mediante la escala de dolor de la Universidad de Melbourne, iniciando 30 minutos posteriores a la extubación endotraqueal del paciente, para luego tomar datos cada 30 minutos hasta completar un período de seis horas. El análisis estadístico se realizó con un test de Kruskal-Wallis para los datos no paramétricos. Los resultados obtenidos mostraron que los puntajes en el grupo tramadol presentaron diferencias estadísticamente significativas $(\mathrm{P}<0,05)$ en el tiempo $0,51,524$ y 5 evidenciando un aumento en la escala del dolor en comparación con el grupo morfina/ lidocaína/ketamina. El presente estudio concluyó que la asociación de varios analgésicos en infusión provee mayor efecto analgésico que el uso de tramadol solo en infusión en hembras caninas sometidas a ovariohisterectomía.

\section{REFERENCIAS}

Anis N, S Berry, N Burton, Lodge D. 1983. The dissociative anaesthetics, ketamine and phencyclidine, selectively reduce excitation of central mammalian neurones by N-Methyl-aspartate. Br J Pharmacol 79, 565-575.

Aguado D, J Benito, I Gómez de Segura. 2010. Reduction of the minimum alveolar concentration of isoflurane in dogs using a constant rate of infusion of lidocaine- ketamine in combination with either morphine or fentanyl. Vet $J 29,1-4$.

Borsook D, A LeBel, B Mc Peek. 1999. Tratamiento del Dolor. $1^{\text {ra }}$ ed. Marbán Libros, Madrid, España.

Bravo M, H Bravo, N Daló. 2008. La flunixin meglumina disminuye los signos de dolor perioperatorio en perras sometidas a ovariohisterectomía. Revista científica (Maracaibo) 2,142-147.

Caballero E, F Ahumada. 2002. SNC. Fármacos tranquilizantes. En: Botana L, Landoni F, Jiménez T. Farmacología y Terapéutica Veterinaria. McGraw-Hill Interamericana, Madrid, España, Pp 162-163.

Dyson D. 2008. Perioperative pain management in veterinary patients. Vet Clin Small Anim 38, 1309-1327.
Firth A, S Haldane. 1999. Development of a scale to evaluate postoperative pain in dogs. J Am Vet Med Assoc 214, 651-659.

Flecknell P, A Waterman-Pearson. 2000. Pain Management in Animals. Saunders, China.

Giorgi M, S Del Carlo, G Saccomanni, B Łebkowska-Wieruszewska, V Turini, C Kowalski. 2009. Biopharmaceutical profile of tramadol in the dog. Vet Res Comm 33, 189-192.

Hall L, K Clarke, C Trim. 2001. Principles of sedation, analgesia and premedication. Veterinary anaesthesia. WB Saunders, London, UK, Pp 75-112.

Hardie E, B Hansen, G Carroll. 1997. Behavior after ovariohysterectomy in the dog: what's normal? Appl Anim Behav Sci 51, 111-128.

Hellebrekers L. 2002. Un Enfoque para el Control Eficaz del Dolor en Pequeños Animales, Especies Exóticas y Equinos: Manejo del Dolor en Medicina Veterinaria. Inter-Médica, Buenos Aires, Argentina, Pp 47-49.

Hellyer P. 2002. Objective, Categoric Methods for Assessing Pain and Analgesia. In: Gaynor J, Muir W (eds). Handbook of Veterinary Pain Management. Mosby, St. Louis, Missouri, USA, Pp 82-107.

Hellyer P, R Downing, J Hagedorn, S Robertson. 2007. AAHA/AAFP Pain management guidelines for dogs. J Am Anim Hosp Assoc 43, 235-248.

Holton L, E Scott, A Nolan, J Reid, E Welsh. 1998. Investigation of the relationship between physiological factors and clinical pain in dogs scored using a numerical rating scale. J Small Anim Pract 39, 469-474.

Holton L, J Reid, E Scott, P Pawson, A Nolan. 2001. Development of a behaviour-based scale to measure acute pain in dogs. Vet Rec 148, 525-531.

Johnson E, T Kamilaris, G Chrousos, P Gold. 1992. Mechanisms of stress: a dynamic overview of hormonal and behavioral homeostasis. Neurosci Biobehav Rev 16, 115-130.

Kongara K, P Chambers, C Johnson. 2009. Glomerular filtration rate after tramadol, parecoxib and pindolol following anaesthesia and analgesia in comparison with morphine in dogs. Vet Anaesth Analg 36, 86-94.

Koppert W, M Weigand, F Neumann, R Sittl, J Schuettler, M Schmelz, W Hering. 2004. Perioperative intravenous lidocaine has preventive effects on postoperative pain and morphine consumption after major abdominal surgery. Anesth Analg 98, 1050-1055.

Kukanich B, M Papich. 2004. Pharmacokinetics of tramadol and the metabolite o-desmethyltramadol in dogs. $J$ Vet Pharmacol Ther 27, 239-246.

Lamont L, W Tranquilli, K Grimm. 2000. Physiology of pain. Vet Clin North Am Small Anim Pract 30, 703-728.

Lascelles B. 2000. Clinical pharmacology of analgesic agents. In: Hellebrekers L (ed). Animal Pain. A practice-oriented approach to an effective pain control in animals. Van Der Wees Uitgeverij, Utrecht, Holland, Pp 85-116.

Lewis K, N Han. 1996. Tramadol: A centrally acting analgesic. Am J Health-Syst Pharm 54, 643-652.

Lucas A, A Firth, G, Anderson, J Vine, G Edwards. 2001. Comparison of the effects of morphine administered by constant-rate intravenous infusion or intermittent intramuscular injection in dogs. $J$ Am Vet Med Assoc 6, 884-891.

Mastrocinque S, D Fantoni. 2003. A comparison of preoperative tramadol and morphine for the control of early postoperative pain in canine ovariohysterectomy. Vet Anaesth Analg 30, 220-228.

McMillan C, A Livingston, C Clark, P Dowling, S Taylor, T Duke, R Terlinden. 2008. Pharmacokinetics of intravenous tramadol in dogs. Rev Canad Rech Vet 72, 325-331.

Morton C, J Reid, E Scott, L Holton, A Nolan. 2005. Application of a scaling model to establish and validate an interval level pain scale for assessment of acute pain in dogs. Am J Vet Res 66, 2154-2166.

Muir W. 2002. Pain and Stress. In: Gaynor J, Muir W (ed). Veterinary Pain Management. Mosby, USA, Pp 46-59.

Muir W, A Wiese, P March. 2003. Effects of morphine, lidocaine, ketamine and morphine-lidocaine-ketamine drug combination on minimum alveolar concentration in dogs anesthetized with isoflurane. Am J Vet Res 64, 1155-1160. 
Nolan A. 2002. SNC. Opioides. En: Botana L, Landoni F, Jiménez T. Farmacología y Terapéutica Veterinaria. McGraw-Hill Interamericana, Madrid, España, Pp 176-177.

Oncken A, R Kirby, E Rudloff. 2001. Hypothermia in critically ill dogs and cats. Compendium 23, 506-521.

Otero P. 2004. Drogas analgésicas En: Otero P. (ed). Dolor: Evaluación y Tratamiento en Pequeños Animales. Inter-médica, Buenos Aires, Argentina, Pp 89-90.

Pekoe G, D Smith. 1982. The involvement of opiate and monoaminergic neuronal systems in the analgesic effects of ketamine. Pain 12, 57-73.

Raffa R, E Friderichs, W Reimann, R Shank, E Codd, J Vaught. 1993. Complementary and synergistic antinociceptive interaction between the enantiomers of tramadol. J Pharmacol Exp Ther 267, 331-340.

Raposo E, A Rodrigues, H Quirilos, D Campagnol, J Gomes. 2009. Comparative study on the sedative effects of morphine, methadone, butorphanol or Tramadol, in combination with acepromazine, in dogs. Vet Anaesth Analg 36, 25-33.

Reza M, C Egger, B Rohrbach, S Cox, T Doherty. 2009. Effects of tramadol on the minimum alveolar concentration of sevoflurane in dogs. Vet Anaesth Analg 36, 334-340.

Sarrau S, J Jourdan, F Dupuis-Soyris, P Verwaerde. 2007. Effects of postoperative ketamine infusion on pain control and feeding behaviour in bitches undergoing mastectomy. J Small Anim Pract 48, 670-676.
Scott L, C Perry. 2000. Tramadol. A review of its use in perioperative pain. Drugs 60, 139-176.

Smith L, E Bentley, A Shih. 2004. Systemic lidocaine infusion as an analgesic for intraocular surgery in dogs: a pilot study. Vet Anaesth Analg 31, 53-63.

Vaisanen M, M Raekallio, E Kuusela, P Huttunen, J Leppaluoto, P Kirves, O Vainio. 2002. Evaluation of the perioperative stress response in dogs administered medetomidine or acepromazine as part of the preanesthetic medication. Am J Vet Res 63, 969-975.

Vickers M, D Paravicini. 1995. Comparison of tramadol with morphine for post-operative pain following abdominal surgery. Eur $J$ Anaesthesiol 12, 1-7.

Wagner A, J Walton, P Hellyer. 2002. Use of low doses of ketamine administered by constant rate infusion as an adjunct for postoperative analgesia in dogs. $J$ Am Vet Med Assoc 221, 72-75.

Wiese A, W Muir, T Wittum. 2005. Characteristics of pain and response to analgesic treatment in dogs and cats examined at a veterinary teaching hospital emergency service. J Am Vet Med Assoc 226, 2004-2009.

Yazbek K, D Fantoni. 2005. Evaluation of tramadol, an "atypical" opioide analgesic in the control of immediate postoperative pain in dogs submitted to orthopedic surgical procedures. Braz J Vet Res Anim Sci 42, 250-258. 